\title{
Implicit priming of conflicting motivational orientations in heavy drinkers
}

Samantha Baker ${ }^{1,2}$, Joanne M Dickson ${ }^{1}$ and Matt Field ${ }^{1,3^{*}}$

\begin{abstract}
Background: Approach and avoidance motivation for alcohol are relatively independent, and they operate in both automatic (or implicit) and controlled processes. In this study, we adapted methods previously used in the appetite literature and implicitly primed an alcohol-related motivational orientation (approach or avoidance) in order to investigate its influence on the opposing motivational orientation, in a group of non-dependent heavy drinkers.

Methods: Participants $(N=110)$ completed computerised measures of attentional bias and avoidance for alcohol cues (visual probe task) and behavioural approach and avoidance for alcohol cues (Stimulus-response Compatibility (SRC) task). Word primes were subliminally presented during each trial of these tasks. Participants were randomly allocated to groups that were exposed to alcohol-appetitive primes, alcohol-aversive primes, or neutral primes.

Results: Contrary to hypotheses, word primes had no effect on responding during the visual probe or SRC tasks. Supplementary analyses revealed that participants showed attentional avoidance of alcohol cues combined with slower behavioural avoidance responses to alcohol cues. Attentional bias was positively correlated with scores on the Alcohol Use Disorders Identification Test.

Conclusions: In contrast to previous findings from the appetite domain, our findings suggest that automatic alcohol cognitions are unaffected by implicit priming of motivational orientations, although features of our novel methodology may account for these results.
\end{abstract}

Keywords: Alcohol, Ambivalence, Approach tendencies, Attentional bias, Implicit cognition, Priming

\section{Background}

Motivational conflict (or ambivalence) arises when people experience two goals that are incompatible with each other. The ambivalence model of craving (Breiner et al. 1999; McEvoy et al. 2004) proposes that the decision to consume alcohol depends on the balance between motivation to indulge ('approach', e.g., wanting to drink at a party) and motivation to refrain ('avoidance', e.g., not wanting to drink at the party because of work commitments the next day). Therefore, alcohol use should vary according to which system is more activated at any one time (Ostafin et al. 2003). Evidence to support the concept of separable approach and avoidance motivation exists in the domains of alcohol use (McEvoy et al. 2004) as well as

\footnotetext{
* Correspondence: mfield@liv.ac.uk

'Department of Psychological Sciences, University of Liverpool, L69 7ZA Liverpool, UK

${ }^{3}$ UK Centre for Tobacco and Alcohol Studies (UKCTAS), Liverpool, UK

Full list of author information is available at the end of the article
}

dieting (Stroebe et al. 2008) and mental health (Dickson and MacLeod 2004).

Multiple factors are known to influence the relative strength of alcohol approach and avoidance motivation, both of which can operate in automatic and controlled processes. Automatic (or implicit) processes are activated spontaneously, are difficult to control, and the individual may be unaware of their operation. On the other hand, controlled (or explicit) processes are rule-based, reflective, and they operate within conscious awareness (Stacy and Wiers 2010). Dual-process theories suggest that both automatic and controlled processes play an important role in the development and maintenance of alcohol use disorders (Wiers et al. 2007). It is suggested that initial alcohol involvement is the result of a controlled process, in which drinkers weigh up the pros and cons of drinking versus not drinking. However, with extensive experience of drinking, automatic processes are strengthened and they come to control drinking behaviour, eventually over-

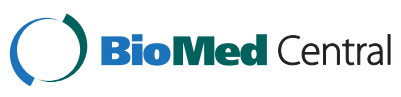

(c) 2014 Baker et al.; licensee BioMed Central Ltd. This is an Open Access article distributed under the terms of the Creative Commons Attribution License (http://creativecommons.org/licenses/by/4.0), which permits unrestricted use, distribution, and reproduction in any medium, provided the original work is properly credited. The Creative Commons Public Domain Dedication waiver (http://creativecommons.org/publicdomain/zero/1.0/) applies to the data made available in this article unless otherwise stated. 
riding conscious intentions to stop drinking (Ostafin et al. 2008). Other theoretical models based on brain adaptations (Robinson and Berridge 1993) make similar predictions: after repeated alcohol use, automatic motivational processes are strengthened and they influence drinking behaviour irrespective of the subjective benefits or costs of drinking.

Researchers within the addiction field have focussed on three types of implicit cognitions: attentional bias, approach tendencies, and spontaneous memory associations. Attentional bias occurs when an individual shows increased attention for particular stimuli (e.g. pictures of alcohol) compared to alternative stimuli, (e.g. pictures of furniture). There are several ways to measure attentional bias, and one of the most commonly used is the visual probe task (Field et al. 2004). In a typical version of this task, two matched pictures are presented on a computer screen for a brief period, before a visual probe replaces one of the pictures. The participant's task is to respond to the probe as quickly as possible. In such a task involving alcohol and neutral pictures, attentional bias would be inferred if the participant was faster to react to probes that replaced alcohol pictures (congruent trials), rather than probes that replaced neutral pictures (incongruent trials). If, however, this pattern were reversed (i.e. participants were faster to react on incongruent trials), this would suggest attentional avoidance of alcohol cues. Several studies have shown that heavy drinkers who are not seeking treatment have an attentional bias for alcohol cues (Field et al. 2004; Townshend and Duka 2001), whereas alcohol-dependent participants who are tested in treatment contexts show initial attentional bias (when pictures are presented very briefly, e.g. 50-100 ms), that is followed by attentional avoidance (when pictures are presented for longer periods of time, upwards of $500 \mathrm{~ms}$ ) (Field et al. 2013; Noël et al. 2006; Stormark et al. 1997; Townshend and Duka 2007; Vollstädt-Klein et al. 2009). This mixed pattern of attentional bias may reflect automatic 'ambivalence' in alcohol dependent participants, with appetitive motivational processes mapping on to the initial attentional bias and aversive motivational processes mapped on to the subsequent attentional avoidance (see Field et al. 2013, for discussion).

Automatic approach and avoidance tendencies elicited by alcohol-related cues have been assessed with the alcohol-related stimulus-response compatibility (SRC) task (Field et al. 2008) and related tasks (Wiers et al. 2009). In the alcohol SRC task, a manikin is presented on a computer screen either above or below an alcoholrelated or neutral picture. Participants must move the manikin towards or away from the pictures as quickly as possible. On some blocks of the task, participants must make the manikin move towards alcohol pictures and away from neutral pictures, whereas these instructions are reversed in other blocks of the task. Automatic approach tendencies for alcohol cues are inferred if participants are faster to respond on blocks of the task when alcohol pictures require the 'approach' movement in comparison to blocks when alcohol pictures require the 'avoidance' movement. By contrast, if participants are faster on the 'avoid alcohol' blocks compared to the 'approach alcohol' blocks, this would suggest that alcohol cues evoke automatic avoidance tendencies. Any observed approach or avoidance bias reflects an automatic process because, although participants are explicitly instructed to approach and avoid different types of pictures as quickly as possible, differences in the speed of responding during 'approach alcohol' and 'avoid alcohol' blocks are not under intentional control (see Watson et al. 2012, for a review). Heavy drinkers who are not seeking treatment display automatic approach tendencies for alcohol cues (Christiansen et al. 2012; Field et al. 2011; Field et al. 2008; Kersbergen et al. 2014). Two studies that administered the task to alcohol-dependent patients in treatment settings reported conflicting findings: no reliable bias in either automatic approach or avoidance evoked by alcohol cues was reported in one study (Barkby et al. 2012) whereas faster avoidance rather than approach, which was predictive of subsequent relapse, was reported in another study (Spruyt et al. 2013). One reason for these findings with alcohol-dependent patients is that the standard version of the SRC task yields an index of automatic approach that is relative to avoidance, therefore the pattern that is observed in heavy drinkers who are not seeking treatment (Christiansen et al. 2012; Field et al. 2011; Field et al. 2008) can be attributed to either strong automatic approach, weak automatic avoidance, or a combination of the two. Among alcohol-dependent patients, if alcohol cues simultaneously evoke strong automatic approach at the same time as strong automatic avoidance, this may explain why this group display either no reliable bias on the standard version of the task (Barkby et al. 2012) or a bias to faster avoidance (Spruyt et al. 2013) depending on the strength of their motivational orientations to avoid alcohol at the time of testing.

Our goal in the present study was to experimentally manipulate alcohol approach and avoidance motivational orientations using a subliminal priming procedure, in order to investigate their influence on automatic attentional biases and approach and avoidance tendencies. Previous research into the cognitive processes that are involved in dietary restriction has shown that a goal state can be primed subliminally (below the threshold of conscious awareness), and this has the effect of suppressing cognitive processing of information related to the conflicting goal state (Stroebe et al. 2008). In two experiments, participants who were attempting to restrict their food intake (in order to lose weight) completed a 
masked priming task in which words were briefly presented and then masked so that they did not cross the threshold of conscious awareness. Primes were either neutral words or words that were intended to activate the goal of eating enjoyment, such as 'tasty' or 'chocolate'. Immediately after this, target words related to either the conflicting goal (dieting), neutral words, or non-words (random letter strings) were presented, and participants were required to rapidly identify whether a word or a non-word was presented. The primary finding was that participants who had been subliminally primed with words related to eating enjoyment were subsequently slower to identify target words that were related to dieting. The authors interpreted their findings as indicating that subliminal priming of one goal (eating enjoyment, in this case), led to a suppression of the competing goal (dieting in order to lose weight, in this case), which manifested as slower identification of diet-related words.

In the current study we aimed to modify and extend the work of Stroebe et al. (Stroebe et al. 2008) and explore the effect of subliminally priming one motivational orientation on the opposing motivational orientation, in relation to automatic cognitive processing biases for alcohol cues. Participants completed measures of attentional bias and automatic approach tendencies whilst their motivational orientation was subliminally primed on a betweensubjects basis. We opted for a between-subjects design instead of a within-subjects design (in which motivational orientation could be primed on a trial-by-trial basis) in order to maximise the potency of the priming intervention and to eliminate the risk of carryover effects from one trial to the next (Brooks 2012). Our primary hypotheses were that, in relation to participants who were subliminally primed with neutral words, participants who were subliminally primed with alcohol-appetitive words would show elevated attentional bias for alcohol pictures that were presented for $50 \mathrm{~ms}$ and $500 \mathrm{~ms}$, and increased automatic approach tendencies and reduced automatic avoidance tendencies. In participants who were subliminally primed with alcohol-aversive words, we predicted the complete opposite pattern of results to that expected for the alcohol-appetitive group. We also investigated the relationships between automatic alcohol cognitions, their counterparts in controlled (self-reported) processes, and individual differences in drinking behaviour and hazardous drinking, in an attempt to replicate previous demonstrations of associations between these constructs (Barkby et al. 2012; Christiansen et al. 2012; Field et al. 2005; Field et al. 2009).

\section{Methods}

\section{Participants}

One hundred and ten heavy drinkers (41 male, 69 female, mean age, $M=32.54, S D=8.01$ ) were recruited from various sites in the North West of England (e.g., local businesses, the University campus, and hospitals). Our sample size was informed by Ostafin \& Brooks (2011) who reported a small-to-medium effect size (Cohen's $d=0.28$ ) for the effects of negative mood induction on automatic alcohol approach tendencies with a total $\mathrm{N}$ of 65 participants, who were divided into two groups. Inclusion criteria were: (i) aged between 25 and 60 years (ii) English as first language, (iii) heavy drinkers (those who drank in excess of UK Department of Health guidelines for 'safe drinking', which are no more than 14 units per week for women and 21 units per week for men; 1 UK unit $=8 \mathrm{~g}$ alcohol), and (iv) normal or corrected-tonormal vision. Exclusion criteria included: (i) current or past alcohol or other substance dependence, (ii) currently suffering from acute mental health difficulties, (iii) positive breath alcohol level. The study was approved by the University of Liverpool Research Ethics Committee and the National Health Service (NHS) Research Ethics Committee (because some participants were NHS staff recruited from NHS premises). All participants provided written informed consent to take part in the study.

Participants were randomly allocated to one of three groups: alcohol-appetitive, alcohol-aversive, or control. Groups did not differ significantly in terms of gender ratio, $\chi^{2}=3.92, p=.14$, highest level of education achieved, $\chi^{2}=.70, p=.95$, or current employment status, $\chi^{2}=6.33$, $p=.18$. Table 1 shows variables related to alcohol consumption and scores on the Alcohol Use Disorders Identification Test (Saunders et al. 1993) and subscales of the Approach and Avoidance of Alcohol Questionnaire (McEvoy et al. 2004). Possible pre-existing differences between groups (alcohol-appetitive, alcohol-aversive, control) on these variables were investigated using a series of One-Way ANOVAs, but there were no significant group differences ( $\mathrm{ps}>.1$ ).

\section{Materials \\ Approach and Avoidance of Alcohol Questionnaire (AAAQ) - 'right now' version (McEvoy et al. 2004)}

This 14-item questionnaire comprises three subscales. The 'inclined/indulgent' subscale captures mild approach inclinations to drink, the 'obsessed/compelled' subscale captures intense approach inclinations, and the 'resolved/ regulated' subscale captures inclinations to avoid drinking. Respondents are asked to rate how strongly they agree with each item, on a 9-point Likert scale anchored from 0 (not at all) to 8 (very strongly).

\section{Alcohol Use Disorders Identification Test (AUDIT) (Saunders et al. 1993)}

This 10-item self-report questionnaire measures patterns of alcohol consumption that are hazardous to health. 
Table 1 Self-report data

\begin{tabular}{|c|c|c|c|c|c|c|c|c|c|}
\hline & & \multicolumn{2}{|c|}{ Alcohol-appetitive ( $\mathrm{N}=38,15$ Male) } & \multicolumn{2}{|c|}{ Alcohol-aversive ( $\mathrm{N}=36,9$ Male) } & \multicolumn{2}{|c|}{ Control $(\mathrm{N}=36,17$ Male) } & \multirow[b]{2}{*}{$F$} & \multirow[b]{2}{*}{$p$} \\
\hline & & Mean & $S D$ & Mean & $S D$ & Mean & $S D$ & & \\
\hline \multirow[t]{3}{*}{ AAAQ } & Inclined-Indulgent & 4.01 & 2.37 & 4.41 & 2.11 & 4.20 & 2.21 & .30 & .74 \\
\hline & Resolved-Regulated & 1.79 & 1.10 & 2.02 & 1.11 & 2.02 & 1.22 & .50 & .61 \\
\hline & Obsessed-Compelled & 0.89 & 1.29 & 1.33 & 1.46 & 1.21 & 1.84 & .81 & .45 \\
\hline AUDIT & & 9.13 & 4.24 & 11.25 & 4.84 & 9.83 & 3.69 & 2.34 & .11 \\
\hline \multirow[t]{2}{*}{ TLFB } & Units & 42.18 & 29.59 & 42.22 & 24.13 & 44.20 & 36.10 & .05 & .95 \\
\hline & Drinking days & 5.79 & 3.03 & 5.83 & 2.54 & 6.20 & 3.20 & .18 & .84 \\
\hline
\end{tabular}

Note. AAAQ = Approach and Avoidance of Alcohol Questionnaire - Right Now; AUDIT = Alcohol Use Disorders Identification Test; TLFB = Time Line Follow Back (diary of alcohol consumption): Units = Total units consumed in two week period before study ( 1 unit $=8 \mathrm{~g}$ alcohol), Drinking days $=$ Total number of days on which alcohol was consumed in two week period.

The questionnaire is scored from 0 to 40 . Scores of 8 or above indicate hazardous drinking.

\section{Timeline followback (TLFB) retrospective drinking diary (Sobell \& Sobell 1992)}

This is a retrospective diary on which participants record their alcohol consumption over the previous fortnight. Such a relatively short timeframe can be used with little to no loss in the accuracy of the data (Vakili et al. 2008). From this, we calculated the number of drinking days each week, and the total volume of alcohol consumed.

\section{Presentation computer}

All computer tasks were programmed in Inquisit software (Millisecond Software 2006) and were presented on a laptop computer with a 15 -inch monitor that was set to a refresh rate of $60 \mathrm{~Hz}$ (equivalent to one screen refresh every $17 \mathrm{~ms}$ ).

\section{Pictorial stimuli}

We used a set of 10 pairs of alcohol-related and matched control pictures, which were a subset of the picture pairs used in previous studies (Barkby et al. 2012; Jones et al. 2012). Each alcohol picture depicted a close-up of an alcoholic drink or a person holding or consuming an alcoholic drink, and was matched with a perceptually similar picture depicting items of office equipment and stationery, or people interacting with those items. We also used 8 pairs of neutral-neutral pictures, which were neutral pictures drawn from the International Affective Picture System (Lang et al. 2008). All pictures measured $95 \mathrm{~mm} \times$ $130 \mathrm{~mm}$ when presented on the screen.

\section{Masked priming stimuli and procedure}

We selected thirty prime words (see Table 2) for use in the visual probe and SRC tasks. These were 10 alcoholappetitive words, (e.g. 'party'), 10 alcohol-aversive words, (e.g. 'nausea'), and 10 neutral words, (e.g. 'bookshelf'). The process for selecting the words was as follows. We initially created a list of 32 alcohol-appetitive and alcohol-aversive words based on words from the lexicon of alcohol and drug terms published by the World Health Organization (World Health Organisation 1994), and additional words suggested by the authors. In a small pilot study, this list was presented to 22 people (who did not participate in the main study) who were asked to select 20 words that would be most likely to encourage (10 words) and discourage (10 words) them from drinking alcohol. The 10 most frequently endorsed words in each category were selected for use in the study. Finally, a list of 10 neutral words relating to items of furniture was created. This category was selected due to its neutral emotional valence and lack of conflict with the neutral and control pictures presented in the computer tasks. All three categories of primes were matched on number of letters and syllables.

The masked priming procedure was embedded at the start of each trial of the SRC and visual probe tasks. The procedure that we adopted was based on our review of the masked priming literature (Degner et al. 2007; Frings and Wentura 2003; Klauer and Musch 2003; Kouider and Dehaene 2007; Moors et al. 2010; Van den Bussche et al. 2009; Wentura and Degner 2010). Firstly, participants were instructed to focus on a forward mask, in the

Table 2 Appetitive, aversive and neutral prime word used in subliminal priming

\begin{tabular}{ccc}
\hline Appetitive & Aversive & Neutral \\
\hline Confident & Dehydrated & Piano-stool \\
Sociable & Hangover & Recliner \\
Going-out & Aggression & Cabinet \\
Celebrate & Embarrassed & Dining-chair \\
Unwind & Nausea & Drawer \\
Relaxed & Depressed & Bookcase \\
Dancing & Headache & Wardrobe \\
Party & Vomit & Table \\
Fun & Tired & Desk \\
Pub & Sick & Bed \\
\hline
\end{tabular}


form of a string of 10 random letters ('pfrvhnqszxykwmg') which was presented on the screen for $500 \mathrm{~ms}$. This was followed by the prime word, with random letters placed either side in order to make it the same length as the mask letter strings, for $34 \mathrm{~ms}$. Finally a backward mask ('gmwkyxzsqnhvrpf') was presented for $51 \mathrm{~ms}$. Therefore, the stimulus onset asynchrony (SOA) was $85 \mathrm{~ms}$. Previous results suggest that these timings are optimal for priming effects (Greenwald et al. 1996; Hermans et al. 2003; Wentura and Degner 2010; Wentura et al. 2005). Immediately afterwards, the target stimuli were presented (see below), and we applied a response window in line with recommendations for maximising masked priming effects (Draine and Greenwald 1998). Due to the nature of the visual probe and SRC tasks, the length of the response window varied between tasks, as detailed below.

\section{Visual probe task}

This is an established tool for the measurement of attentional biases for substance-related cues in substance users (Field et al. 2004; Townshend and Duka 2001). On each trial, following presentation of the forward mask, prime and backward mask (as detailed above), a pair of pictures was presented on the left and right hand sides of the computer screen for either $50 \mathrm{~ms}$ or $500 \mathrm{~ms}$. Immediately after picture offset, a small dot probe appeared on either the left or right hand side of the screen, to which participants were required to respond by pressing a key on the left or right hand side of the keyboard, as quickly as possible and within a response window of $500 \mathrm{~ms}$. In addition to trials with alcohol and matched control pictures, we also included 'neutral-neutral' trials in which a pair of neutral pictures was presented; these trials were included in order to distinguish biases in attentional orienting and attentional disengagement on the primary (alcoholcontrol) trials (Koster et al. 2004).

The task consisted of two blocks, one in which picture pairs were presented for $50 \mathrm{~ms}$ and another in which picture pairs were presented for $500 \mathrm{~ms}$. Block order was counterbalanced across group. Each block comprised 10 practice and buffer trials, in which neutral-neutral picture pairs were presented, followed by 100 critical trials, comprising 80 alcohol-control trials and 20 neutral-neutral trials, which were presented in a new random order for each participant. On the alcohol-control trials, each picture pair was presented eight times: four times with the alcohol picture on the left, and four times with the alcohol picture on the right, and within this the probe replaced alcohol and control pictures with equal frequency. There was an intertrial interval of $1000 \mathrm{~ms}$, and the task took approximately ten minutes to complete. Reaction times to the probes and errors (pressing the wrong button or failing to respond within the response window) were recorded.

\section{Stimulus-response Compatibility (SRC) task}

Participants were required to categorise alcohol-related and control pictures by moving a manikin (matchstick man) towards or away from the pictures as quickly as possible. The standard version of the task (Field et al. 2008; Field et al. 2005) is split into two blocks: in one block, participants must 'approach' the alcohol pictures and 'avoid' the control pictures. In the other block, these instructions are reversed. If participants are faster to respond on the 'approach alcohol' block compared to the 'avoid alcohol' block, this suggests that alcohol cues activate automatic approach tendencies, the pattern that is generally seen in heavy, non-dependent drinkers (Christiansen et al. 2012; Field et al. 2011; Field et al. 2008; see Field et al. 2011, for discussion of underlying psychological mechanisms).

This standard version of the task provides an index of automatic approach tendencies that is relative to automatic avoidance tendencies; therefore, it is impossible to ascertain whether the pattern of results reported in heavy drinkers is indicative of strong automatic approach, weak automatic avoidance, or a combination of the two. We modified the task for the present study so that we were able to quantify automatic alcohol approach and avoidance tendencies independently of each other. This version of the task included neutral movements (to the side) in addition to the standard approach and avoidance movements, and was split into four blocks instead of two.

On each trial, following presentation of the forward mask, prime and backward mask (as detailed above), a picture was presented in the centre of the screen and a small manikin was presented either directly above or below the picture. Participants were required to rapidly move the manikin towards the picture (approach movement), away from the picture (avoidance movement) or to the side (neutral movement) depending on task instructions, by pressing one of three keyboard keys that were labelled 'up', 'down' and 'left' (or 'right'). Participants were instructed to respond as quickly as possible and within a response window of $700 \mathrm{~ms}$. Allocation of the sideways movement to left or right keys was counterbalanced across participants across experimental groups.

The task consisted of four blocks of trials, the order of which was counterbalanced across groups. Each block comprised 12 practice trials (not analysed) followed by 56 critical trials. A subset of seven of the alcohol-related and matched stationery pictures described above were used in this task. The four blocks had different instructions: 1) move the manikin towards alcohol-related pictures and to the side for stationery pictures ('approach alcohol' block); 2) move the manikin towards stationery pictures and to the side for alcohol-related pictures ('approach control' block); 3) move the manikin away from alcohol-related pictures and to the side for stationery 
pictures ('avoid alcohol' block); 4) move the manikin away from stationery pictures and to the side for alcohol-related pictures ('avoid stationery' block). Correct responses caused the manikin to move in the appropriate direction in a short animation that lasted $500 \mathrm{~ms}$; incorrect responses led to the presentation of a large red ' $\mathrm{X}$ ' in the centre of the screen for $500 \mathrm{~ms}$. In each block, each picture was presented four times each, twice with the manikin above and twice with the manikin below the picture. Trials were presented in a random order within each block. There was an intertrial interval of $1000 \mathrm{~ms}$, and the task took approximately 15 minutes to complete. Reaction times to initiate movement of the manikin and errors (pressing the wrong button or failing to respond within the response window) were recorded.

\section{Prime visibility measure}

We devised a forced choice recognition task to check participants' awareness of the word primes that were presented during the masked priming procedure. This is an established method for evaluating prime visibility (Degner et al. 2007). On each trial of the task, a masked prime word was individually presented in the centre of the screen under exactly the same conditions as those applied during the SRC and visual probe tasks (i.e. with a forward mask for $500 \mathrm{~ms}$, prime word for $34 \mathrm{~ms}$ and finally a backward mask for $51 \mathrm{~ms}$ ). Following this, two prime words were displayed on screen, one of which was the prime word previously displayed (i.e. the target) and another was a different prime word. Participants were asked to press the left or right key on the keyboard to indicate which word they thought they had seen. Participants completed 20 trials of this forced recognition test; the primes presented were the same as those shown during the SRC and visual probe tasks (either alcohol-appetitive, alcohol-aversive, or neutral). No timeout was applied, so participants could take as long as they wished to respond on each trial. The task took less than two minutes to complete. The dependent variable was the percentage of prime words that were correctly identified, and we anticipated that participants would respond at chance levels, i.e. with $50 \%$ accuracy.

\section{Procedure}

Participants were tested individually in a quiet, private room at their place of work. After providing informed consent, participants were breathalysed using a Lion Alcolmeter (Lion Laboratories, Barry, UK) to confirm a zero breath alcohol level. Participants then provided demographic information before the laptop computer was set up exactly $50 \mathrm{~cm}$ in front of them. Participants then completed the SRC and visual probe tasks; the order of task completion was counterbalanced across groups. Verbal instructions emphasised the importance of rapid responding, and the experimenter did not mention the word primes. Participants then completed the prime visibility measure before completing the AAAQ, AUDIT and TLFB questionnaires. The entire session took approximately 45 minutes, and participants were thoroughly debriefed at the end of the study. Participants received a $£ 10$ shopping voucher as compensation for their travel expenses and time.

\section{Data analysis}

Given that a strict response window was applied on the SRC and visual probe tasks, we anticipated a higher rate of errors compared to the standard versions of the task in which either a very relaxed (typically at least $1 \mathrm{~s}$ ) response window is applied, or no response window is applied at all. Box and whisker plots of the error data for both tasks suggested that up to $40 \%$ errors could be permitted before participants should be excluded from analyses due to an outlying error rate. This resulted in the loss of 22 participants' data from the SRC task, eight from the 50 ms block of the visual probe task and six from the $500 \mathrm{~ms}$ block of the visual probe task. For the remaining participants, the mean number of errors on the tasks was $19 \%$ for the SRC task, $9 \%$ for the $50 \mathrm{~ms}$ visual probe task and $5 \%$ for the $500 \mathrm{~ms}$ visual probe task.

For the remaining trials, we excluded data from individual trials if response latencies were faster than $100 \mathrm{~ms}$. To deal with outliers, data were discarded on trials when reaction times exceeded the participants' mean reaction times by three standard deviations. For participants retained in the analyses, on average $22 \%$ of data was lost for the SRC task, $10 \%$ for the $50 \mathrm{~ms}$ visual probe task, and $6 \%$ for the $500 \mathrm{~ms}$ visual probe task due to errors and outliers combined.

\section{Results}

\section{Prime visibility check}

Participants completed 20 trials of the forced recognition task. The range of correct responses was 5 to 17 $(M=10.32, S D=2.55)$. A one-sample $t$-test indicated that performance on this task was at chance levels, i.e. not significantly different from 10 , the $50 \%$ level $t(110)=1.3$, $p>$.20. Furthermore, only three participants (one from each group) were correct on sixteen or more trials (more than two standard deviations above the sample mean), and at the end of the task participants were asked if they had been able to see the prime words, and all participants indicated that they could not see the words and they had been guessing during the prime visibility task. However, there was a significant group difference in accuracy on the prime visibility check $\left(F(2,110)=3.54, \quad p=.033, \quad \eta_{\mathrm{p}}^{2}=.06\right)$. Although this difference appears to reflect superior accuracy in the 
control group ( $M=11.25$ correct trials) compared to both alcohol-appetitive and alcohol-aversive groups, who did not differ from each other (both $M=10.00$ correct trials), post-hoc Bonferroni contrasts revealed that the differences between control and alcohol-appetitive $(p=.066)$ and alcohol-aversive $(p=.072)$ groups were not statistically significant.

\section{Visual probe task (Figures 1 and 2)}

We performed a $3 \times 2 \times 3$ mixed ANOVA with group (alcohol-appetitive, alcohol-aversive and control) as the between-subjects factor and task block $(50 \mathrm{~ms}$ and $500 \mathrm{~ms}$ ) and trial type (incongruent, congruent and neutral-neutral) as within-subjects factors. The hypothesised group $\times$ trial type interaction was not significant $\left(\mathrm{F}(4,192)=1.31, \mathrm{p}=.27, \boldsymbol{\eta}_{\mathrm{p}}^{2}=.03\right)$. There was a significant main effect of task block $F(1,96)=9.78, p=.002$, $\boldsymbol{\eta}_{\mathrm{p}}^{2}=.09$, which was subsumed under a significant task block $\mathrm{x}$ trial type interaction $F(2,182)=4.57, p=0.014$, $\boldsymbol{\eta}_{\mathrm{p}}^{2}=.05$. The 3 way interaction group $\mathrm{x}$ trial type $\mathrm{x}$ task block was also not statistically significant $(F(4,192)=.45$, $\left.p=.76, \boldsymbol{\eta}_{\mathrm{p}}^{2}=.01\right)$, and there were no other significant main effects or interactions $(F s<1.26, p s>.2)$.

We conducted paired samples t-tests to investigate the interaction. These revealed that on the 50 ms block, participants were significantly faster to respond on incongruent trials compared to both congruent trials, $t(101)=2.43$, $p=.017$, and neutral-neutral trials, $t(101)=3.12, p=.002$. Therefore, all participants showed attentional avoidance of alcohol pictures presented for $50 \mathrm{~ms}$, which was contrary to expectations. On the $500 \mathrm{~ms}$ block, there were no significant differences between trial types (all $p \mathrm{~s}>.05$ ).

\section{SRC task (Figure 3)}

Mean reaction times during each block of the task were also analysed using a mixed design ANOVA with response type (approach, avoidance) and picture type (alcohol, control) as the within-subjects factors and group as the between-subjects factor. The hypothesised group $\mathrm{x}$ response type $\mathrm{x}$ picture type interaction was not statistically significant $\left(F(1,85)=1.30, p=.28, \boldsymbol{\eta}_{\mathrm{p}}^{2}=.03\right)$.

However, there was a significant interaction between response type and picture type $(F(1,85)=6.16, p=.015$, $\left.\eta_{\mathrm{p}}^{2}=.07\right)$. Paired samples t-tests revealed that participants tended to be faster to approach rather than avoid alcohol pictures, although this difference only approached significance $(t(87)=1.86, p=.07)$, but latencies to approach and avoid control pictures did not differ $(t(87)=1.23, p=.22)$. Furthermore, comparisons between categorisation latencies for alcohol and control pictures demonstrated that participants tended to be slower to avoid alcohol rather than control pictures (again, this difference only approached significance; $t(87)=1.86, p=.07$ ), but latencies to approach alcohol and control pictures did not differ $(t(87)=1.07, p=.29)$.

There was also a significant interaction between response type and group $\left(F(2,85)=3.71, p<.05, \eta_{\mathrm{p}}^{2}=.08\right)$. This interaction was not hypothesised so we did not conduct post-hoc tests in order to unravel it, although inspection of group differences in Figure 3 suggests that participants in the neutral group tended to respond faster on approach blocks relative to avoidance blocks overall. No further significant main effects or interactions were found $(F s<1.23$, ps $>.2$ ).

Finally, we also conducted the same mixed design ANOVA on the error data (frequency of errors during each block of the task). There were no significant main effects or interactions $(F s<2.98, p s>.09)$.

\section{Correlations between variables}

Pearson's correlations were employed to investigate the relationship between attentional bias, approach and avoidance indices from the SRC task, and scores on the three subscales of the AAAQ, alcohol consumption, and scores on the AUDIT. Attentional bias was calculated for each block of the visual probe task $(50 \mathrm{~ms}$ and $500 \mathrm{~ms}$ SOAs) by subtracting mean reaction times on congruent trials (trials where the probe replaces an alcohol picture) from mean reaction times on incongruent trials (where the probe replaces the neutral picture) such that a positive score is indicative of attentional bias for alcohol pictures. On the SRC task, approach bias was calculated by subtracting the mean reaction times on the 'approach alcohol' block from the mean reaction times on the 'approach control' block; avoidance bias was calculated by subtracting the mean reaction times on the 'avoid alcohol' block from the mean reaction time on the 'avoid control' block.

Analysis revealed weak positive correlations between attentional bias on the $500 \mathrm{~ms}$ SOA block and total scores on the AUDIT, $r=.20,(N=104), p($ two-tailed $)=.047$. There were no other significant correlations.

\section{Discussion}

Contrary to expectations, we found no effects of masked priming of motivational orientations on attentional bias for alcohol cues, or on automatic approach and avoidance tendencies evoked by those cues. Given that we developed novel methods for use in this study, we first consider methodological issues that may account for these results, before discussing their theoretical and clinical implications.

Our line of investigation was inspired by research into dietary restraint, another domain in which motivational conflict within both automatic and controlled processes is thought to be important (Stroebe et al. 2013). One study, Stroebe et al. (2008) demonstrated that subliminal 

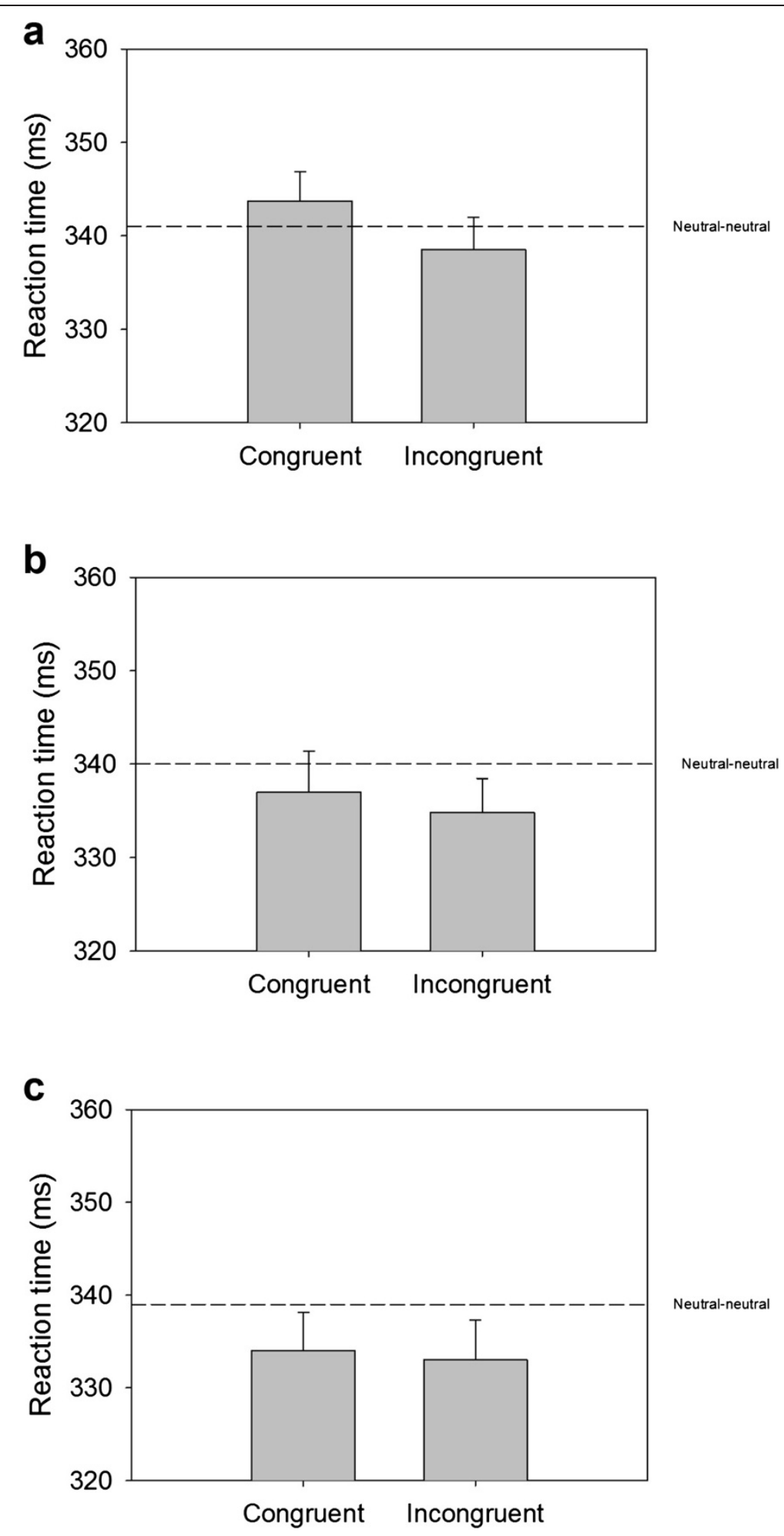

Figure 1 Response times during the 50 ms block of the visual probe task during congruent trials (probe replaces alcohol picture) and incongruent trials (probe replaces neutral picture). The dashed line indicates reaction time on neutral-neutral trials, for comparison. Data from the alcohol-appetitive group are in panel (a), from the alcohol-aversive group in panel (b) and from the control group in panel (c). Values are mean \pm SEM. 

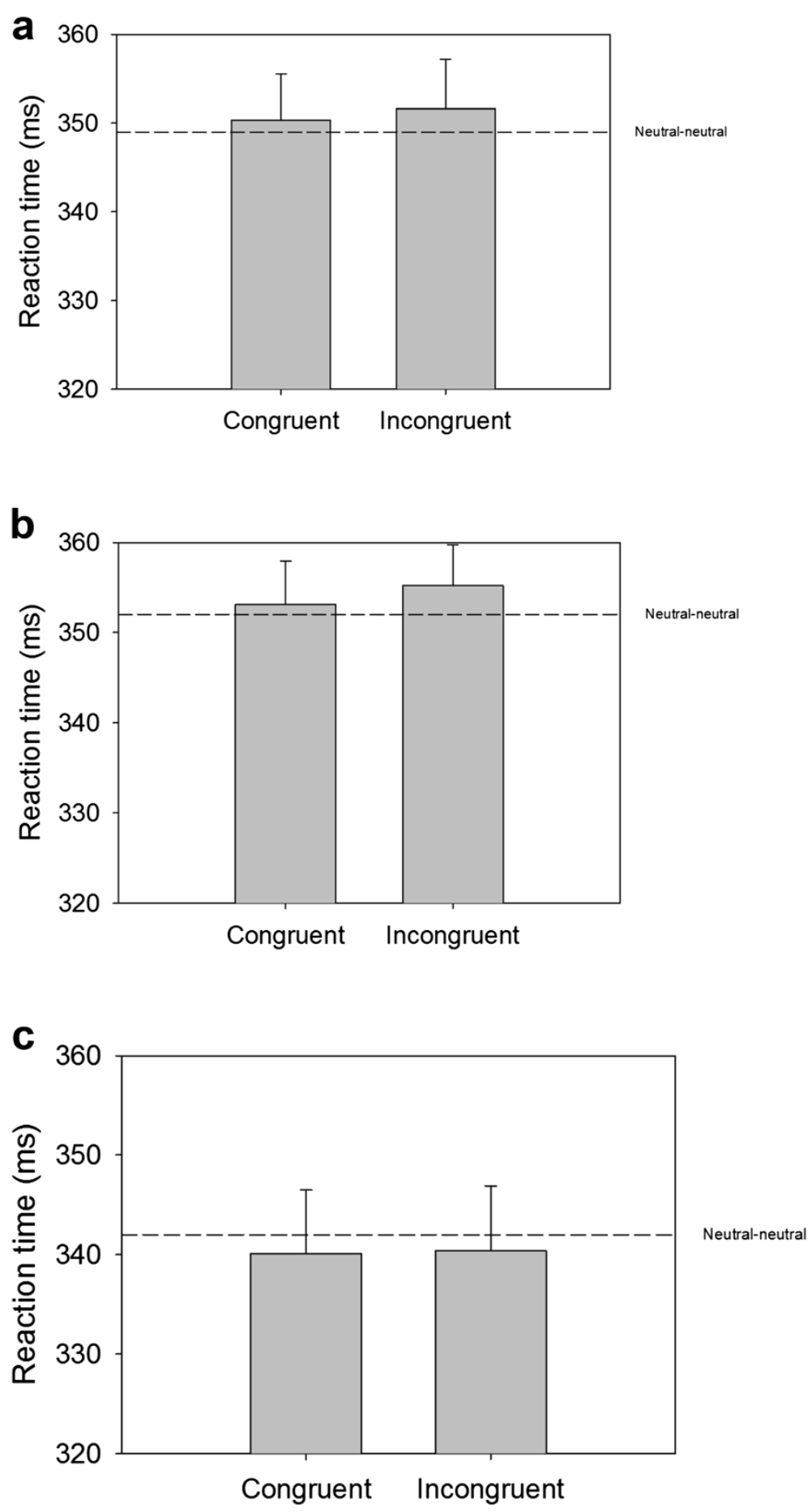

Figure 2 Response times during the $\mathbf{5 0 0}$ ms block of the visual probe task during congruent trials (probe replaces alcohol picture) and incongruent trials (probe replaces neutral picture). The dashed line indicates reaction time on neutral-neutral trials, for comparison. Data from the alcohol-appetitive group are in panel (a), from the alcohol-aversive group in panel (b) and from the control group in panel (c). Values are mean \pm SEM.

priming of one motivational orientation (focussing on the appetising properties of food) led to immediate but transient suppression of a competing motivational orientation (dieting to lose weight). On this basis, we hypothesised that subliminal priming of an alcohol-appetitive motivational orientation would strengthen the automatic appetitive properties of alcohol cues, and weaken the automatic aversive properties of alcohol cues, as indicated by changes 

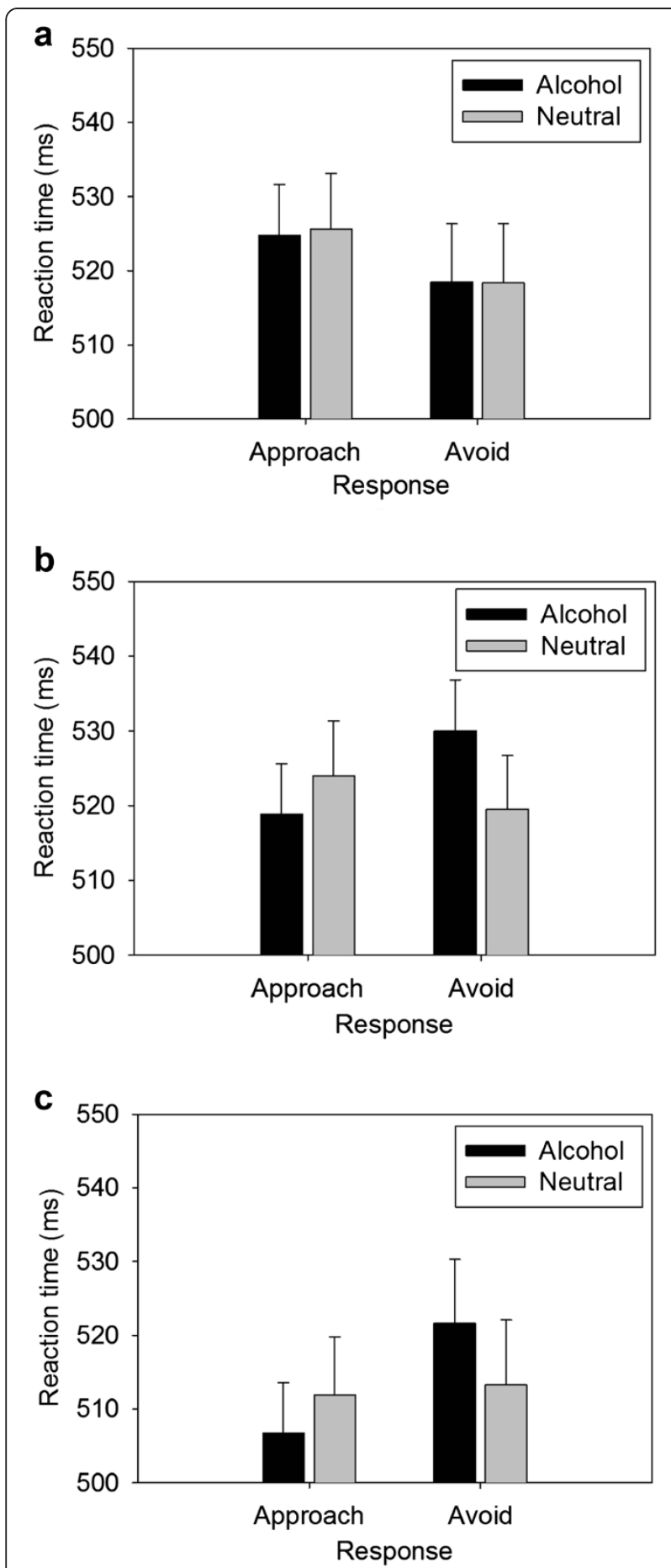

Figure 3 Response times during the modified Stimulus-response Compatibility (SRC) task. The bars indicate sub-blocks of the task, and indicate which stimulus had to be approached or avoided on that sub-block. Data from the alcohol-appetitive group are in panel (a), from the alcohol-aversive group in panel (b) and from the control group in panel (c). Values are mean \pm SEM. in attentional bias and automatic approach and avoidance tendencies evoked by those cues. We also predicted that priming of an alcohol-aversive motivational orientation would lead to exactly the opposite pattern of results. However, our data clearly did not support these hypotheses, because the subliminal priming manipulation had no effect whatsoever on our measures of automatic alcohol cognitions.

In order to interpret these findings, we first consider possible methodological issues. Firstly, no previous studies have incorporated a masked priming procedure within trials of the visual probe and SRC tasks. These aspects of the experimental procedure were implemented following a critical summary of the available literature on masked priming (Greenwald et al. 1996; Hermans et al. 2003; Wentura and Degner 2010; Wentura et al. 2005). The masked priming procedure necessitated presentation of a masked prime before each trial of the visual probe and SRC tasks, together with imposition of a response window at the end of each trial. In hindsight, we suggest that the imposition of a response window, in particular, may have invalidated the primary dependent measure (manual reaction time) from these tasks. In standard versions of these tasks participants are pressured to respond quickly but the response windows applied are normally fairly generous (typically upwards of $1 \mathrm{~s}$ ). The imposition of more stringent response windows in the present study (500 ms for the visual probe task, $700 \mathrm{~ms}$ for the SRC task), while essential for the demonstration of masked priming effects (Draine and Greenwald 1998) may have increased the time pressure on participants to such an extent that this masked the (typically subtle) differences between experimental conditions that are usually seen with these tasks. The high error rates on all tasks (error rates include responses that were too slow), which were expected but were still noticeably higher than those seen when conventional versions of these tasks are used, lend credibility to this argument. For example, the error rate for the modified SRC task used in this study was $19 \%$, as opposed to the 4-6\% error rate that is seen with standard versions of the task that do not apply a response window (Field et al. 2008, 2011; Kersbergen et al. 2014).

Another methodological issue is the inclusion of a sideways (left or right) movement in the SRC task, which was intended as a neutral condition, distinct from approach or avoidance. This modification was informed by previous work demonstrating that the labels given to approach and avoidance movements are more important than the direction of the movements themselves (e.g. Eder and Rothermund 2008). We believe that the specific method used in the present study is an improvement on our first attempt to include a neutral control condition for this task (Barkby et al. 2012). However it is possible that our participants may have encoded the 
sideways movement as an 'avoidance' movement, given that the manikin's starting position was always directly above or below the picture, and therefore the sideways movement involved moving 'away' (see Krieglmeyer et al. 2010). Future research should aim to identify an optimal neutral or control movement in order to distinguish between automatic approach and avoidance tendencies evoked by different cues.

If we are to assume that the present findings cannot be attributed to methodological implications of the tasks used, we must consider the broader theoretical implications of these findings. Prior research has demonstrated that more overt manipulations of the motivational orientation to drink, or to refrain from drinking, have a clear influence on attentional bias for alcohol cues. For example, administration of a 'priming' dose of alcohol leads to increased desire to drink alcohol that is paralleled by an increase in attentional bias for alcohol cues (reviewed in Field et al. 2010). Conversely, if alcohol is made aversive by adulterating the taste of an alcoholic drink with a bittering agent, this leads to a reduction in attentional bias for alcohol cues (Rose et al. 2013). However, the former effects are not consistently seen (Christiansen et al. 2013; Fernie et al. 2012; Miller and Fillmore 2011; Weafer and Fillmore 2013) which is problematic for theoretical models that posit strong inter-relationships between attentional bias and the motivation to drink or consume other addictive substances (Field and Cox 2008; Franken 2003). The effects of experimental manipulation of motivational orientations to drink on various measures of automatic approach and avoidance tendencies have also been investigated, with mixed findings (Christiansen et al. 2013; Fernie et al. 2012; see Watson et al. 2012, for a review). However, overt manipulations of negative mood appear to increase attentional biases (e.g. Field and Powell 2007; Grant et al. 2007) and automatic approach tendencies for alcohol cues, at least in some subgroups of participants (Ostafin and Brooks 2011; Cousijn et al. 2014). Aside from these effects of negative mood, it appears that even very overt manipulations of the motivation to drink or to refrain from drinking do not have particularly robust effects on automatic alcohol cognitions, particularly automatic approach tendencies. To our knowledge, the present study is the first to investigate this issue using a subliminal priming manipulation, and the clear failure of this manipulation should be considered in the context of these previous studies. The overall implication is that automatic alcohol cognitions may be relatively immune to shortterm experimental manipulations of the motivation to drink or to refrain from drinking. Disparate to the domain of dietary restriction (Stroebe et al. 2008) the present study suggests that even subliminal priming of motivational orientations may have no influence on automatic processing of alcohol cues. If confirmed, this would have important clinical implications. For example, automatic appetitive alcohol cognitions are thought to play a causal role in the development and maintenance of alcohol use disorders, and in relapse to heavy drinking after a period of abstinence. Direct manipulation of automatic processes, for example through repeated practice of alcohol-avoidance movements, may be the only way to change the underlying associations (Wiers et al. 2013).

Finally, we note some interesting secondary findings from our study. Participants were slower to avoid alcohol pictures rather than control pictures on the modified SRC task, but latencies to approach alcohol and control pictures did not differ. This has important implications for our understanding of the psychological mechanisms that underlie performance on this and similar measures of automatic approach tendencies, particularly whether any biases reflects strong approach associations or weak avoidance associations, or both (cf. Field et al. 2008; Ostafin et al. 2003). Regarding attentional bias, we found that all participants tended to shift their attention away from alcohol pictures that were presented for $50 \mathrm{~ms}$, but no attentional bias was present when pictures were presented for $500 \mathrm{~ms}$. Neither of these findings were anticipated on the basis of previous studies, which have shown attentional bias for alcohol pictures presented for $500 \mathrm{~ms}$ but no bias for pictures presented for very brief durations in non-dependent heavy drinkers (Field and Cox 2008). However, the previous discussion about the imposition of a response window rendering the reaction time measure of attentional bias invalid should be considered here. Alternatively, participants may have attempted to avoid looking at alcohol cues given that most were tested at a time of day (during the daytime) and location (at their place of work) where drinking alcohol was not permitted or socially acceptable. Future studies of this type should be more mindful of the importance that broad contextual factors may play in the expression of automatic cognitive processing biases. Finally, the observed positive correlation between scores on the AUDIT and attentional bias for alcohol cues at $500 \mathrm{~ms}$ are consistent with previous findings demonstrating that attentional bias for alcohol cues is associated with heavy drinking (Field and Cox 2008).

\section{Conclusions}

We failed to find support for our hypotheses that implicit priming of alcohol-appetitive or alcohol-aversive motivational states would influence attentional biases and approach tendencies evoked by alcohol-related cues. We developed a novel methodology to test our hypotheses and features of the methods used may account for our results. Nonetheless, our results suggest that automatic alcohol cognitions cannot be influenced by subliminal priming 
of motivational orientations, a conclusion that has important theoretical and clinical implications.

\section{Competing interests}

The authors declare that they have no competing interests.

\section{Authors' contributions}

MF and JMD conceived of the study, participated in its design and co-ordination and helped to draft the manuscript. SB designed the study, prepared experimental materials, collected and analysed the data, and drafted the manuscript. All authors read and approved the final manuscript.

\section{Acknowledgements}

This research was funded by a research grant from the Wellcome Trust, reference $086247 / Z / 08 / Z$, awarded to MF and JMD.

\section{Author details}

'Department of Psychological Sciences, University of Liverpool, L69 7ZA Liverpool, UK. ' 2 ancashire Care Foundation Trust, Preston, UK. ${ }^{3}$ UK Centre for Tobacco and Alcohol Studies (UKCTAS), Liverpool, UK.

\section{Received: 12 May 2014 Accepted: 6 August 2014}

\section{Published: 19 August 2014}

\section{References}

Barkby, H, Dickson, JM, Roper, L, \& Field, M. (2012). To Approach or Avoid Alcohol? Automatic and Self-Reported Motivational Tendencies in Alcohol Dependence. Alcoholism: Clinical and Experimental Research, 36(2), 361-368.

Breiner, MJ, Stritzke, WGK, \& Lang, AR. (1999). Approaching avoidance: A step essential to the understanding of craving. Alcohol Research and Health, 23(3), 197-206.

Brooks, JL. (2012). Counterbalancing for serial order carryover effects in experimental condition orders. Psychological Methods, 17, 600-614.

Christiansen, P, Cole, JC, Goudie, AJ, \& Field, M. (2012). Components of behavioural impulsivity and automatic cue approach predict unique variance in hazardous drinking. Psychopharmacology, 219(2), 501-510.

Christiansen, P, Rose, AK, Cole, JC, \& Field, M. (2013). A comparison of the anticipated and pharmacological effects of alcohol on cognitive bias, executive function, craving and ad-lib drinking. Journal of Psychopharmacology, 27(1), 84-92.

Cousijn, J, Luitjen, M, \& Wiers, RW. (2014). Mechanisms underlying alcohol-approach action tendencies: the role of emotional primes and drinking motives. Frontiers in Psychiatry, 5(article 44), 1-9.

Degner, J, Wentura, D, Gniewosz, B, \& Noack, P. (2007). Hostility-related prejudice against turks in adolescents: Masked affective priming allows for a differentiation of automatic prejudice. Basic and Applied Social Psychology, 29(3), 245-256.

Dickson, JM, \& MacLeod, AK. (2004). Approach and avoidance goals and plans: Their relationship to anxiety and depression. Cognitive Therapy and Research, 28(3), 415-432.

Draine, SC, \& Greenwald, AG. (1998). Replicable Unconscious Semantic Priming. Journal of Experimental Psychology: General, 127(3), 286-303.

Eder, AB, \& Rothermund, K. (2008). When do motor behaviors (mis)match affective stimuli? An evaluative coding view of approach and avoidance reactions. Journal of Experimental Psychology: General, 137, 262-281.

Fernie, G, Christiansen, P, Cole, JC, Rose, AK, \& Field, M. (2012). Effects of 0.4 g/kg alcohol on attentional bias and alcohol-seeking behaviour in heavy and moderate social drinkers. Journal of Psychopharmacology, 26(7), 1017-1025.

Field, M, \& Cox, WM. (2008). Attentional bias in addictive behaviors: A review of its development, causes, and consequences. Drug and Alcohol Dependence, 97(1-2), 1-20.

Field, M, \& Powell, H. (2007). Stress increases attentional bias for alcohol cues in social drinkers who drink to cope. Alcohol and Alcoholism, 42, 560-566.

Field, M, Mogg, K, Zetteler, J, \& Bradley, BP. (2004). Attentional biases for alcohol cues in heavy and light social drinkers: The roles of initial orienting and maintained attention. Psychopharmacology, 176(1), 88-93.

Field, M, Mogg, K, \& Bradley, BP. (2005). Craving and cognitive biases for alcohol cues in social drinkers. Alcohol and Alcoholism, 40(6), 504-510.

Field, M, Kiernan, A, Eastwood, B, \& Child, R. (2008). Rapid approach responses to alcohol cues in heavy drinkers. Journal of Behavior Therapy and Experimental Psychiatry, 39(3), 209-218.
Field, M, Munafò, MR, \& Franken, IHA. (2009). A Meta-Analytic Investigation of the Relationship Between Attentional Bias and Subjective Craving in Substance Abuse. Psychological Bulletin, 135(4), 589-607.

Field, M, Wiers, RW, Christiansen, P, Fillmore, MT, \& Verster, JC. (2010). Acute alcohol effects on inhibitory control and implicit cognition: Implications for loss of control over drinking. Alcoholism: Clinical and Experimental Research, 34(8), 1346-1352.

Field, M, Caren, R, Fernie, G, \& De Houwer, J. (2011). Alcohol Approach Tendencies in Heavy Drinkers: Comparison of Effects in a Relevant Stimulus-response Compatibility Task and an Approach/Avoidance Simon Task. Psychology of Addictive Behaviors, 25(4), 697-701.

Field, M, Mogg, K, Mann, B, Bennett, GA, \& Bradley, BP. (2013). Attentional biases in abstinent alcoholics and their association with craving. Psychology of Addictive Behaviors, 27(1), 71-80.

Franken, IHA. (2003). Drug craving and addiction: Integrating psychological and neuropsychopharmacological approaches. Progress in NeuroPsychopharmacology and Biological Psychiatry, 27(4), 563-579.

Frings, C, \& Wentura, D. (2003). Who is watching Big Brother? TV consumption predicted by masked affective priming. European Journal of Social Psychology, 33(6), 779-791.

Grant, W, Stewart, SH, \& Birch, CD. (2007). Impact of positive and anxious mood on implicit alcohol-related cognitions in internally motivated undergraduate drinkers. Addictive Behaviors, 32, 2226-2237.

Greenwald, AG, Draine, SC, \& Abrams, RL. (1996). Three cognitive markers of unconscious semantic activation. Science, 273(5282), 1699-1702.

Hermans, D, Spruyt, A, De Houwer, J, \& Eelen, P. (2003). Affective priming with subliminally presented pictures. Canadian Journal of Experimental Psychology, 57(2), 97-114.

Jones, A, Hogarth, L, Christiansen, P, Rose, AK, Martinovic, J, \& Field, M. (2012). Reward expectancy promotes generalized increases in attentional bias for rewarding stimuli. Quarterly Journal of Experimental Psychology, 65, 2333-2342.

Kersbergen, I, Woud, ML, \& Field, M. (2014). The validity of different measures of automatic alcohol action tendencies. Psychology of Addictive Behaviors, in press.

Klauer, KC, \& Musch, J. (2003). Affective priming: findings and theories. In J Musch \& KC Klauer (Eds.), The psychology of evaluation: Affective processes in cognition and emotion (pp. 7-49). Mahwah, NJ: Lawrence Erlbaum Associates.

Koster, EHW, Crombez, G, Verschuere, B, \& De Houwer, J. (2004). Selective attention to threat in the dot probe paradigm: Differentiating vigilance and difficulty to disengage. Behaviour Research and Therapy, 42(10), 1183-1192.

Kouider, S, \& Dehaene, S. (2007). Levels of processing during non-conscious perception: A critical review of visual masking. Philosophical Transactions of the Royal Society, B: Biological Sciences, 362(1481), 857-875.

Krieglmeyer, R, Deutsch, R, De Houwer, J, \& De Raedt, R. (2010). Being moved: Valence activates approach-avoidance behavior independently of evaluation and approach-avoidance intentions. Psychological Science, 21, 607-613.

Lang, PJ, Bradley, MM, \& Cuthbert, BN. (2008). International affective picture system (IAPS). Affective ratings of pictures and instruction manual. Technical Report A-8. Gainsville, FL: University of Florida.

McEvoy, PM, Stritzke, WGK, French, DJ, Lang, AR, \& Ketterman, RL. (2004). Comparison of three models of alcohol craving in young adults: A cross-validation. Addiction, 99(4), 482-497.

Miller, MA, \& Fillmore, MT. (2011). Persistence of attentional bias toward alcoholrelated stimuli in intoxicated social drinkers. Drug and Alcohol Dependence, $117(2-3), 184-189$.

Millisecond Software. (2006). Inquisit 2.0.60616 (Computer Software). Seattle, WA.

Moors, A, Spruyt, A, \& De Houwer, J. (2010). In search of a measure that qualifies as implicit: Recommendations based on a decompositional view of automaticity. In B Gawronski \& BK Payne (Eds.), Handbook of implicit social cognition: Measurement, theory, and applications (pp. 19-37). New York, NY: The Guilford Press.

Noël, X, Colmant, M, Van Der Linden, M, Bechara, A, Bullens, Q, Hanak, C, \& Verbanck, P. (2006). Time course of attention for alcohol cues in abstinent alcoholic patients: The role of initial orienting. Alcoholism: Clinical and Experimental Research, 30(11), 1871-1877.

Ostafin, BD, \& Brooks, JJ. (2011). Drinking for relief: Negative affect increases automatic alcohol motivation in coping-motivated drinkers. Motivation and Emotion, 35(3), 285-295.

Ostafin, BD, Palfai, TP, \& Wechsler, CE. (2003). The Accessibility of Motivational Tendencies Toward Alcohol: Approach, Avoidance, and Disinhibited Drinking. Experimental and Clinical Psychopharmacology, 11(4), 294-301. 
Ostafin, BD, Marlatt, GA, \& Greenwald, AG. (2008). Drinking without thinking: An implicit measure of alcohol motivation predicts failure to control alcohol use. Behaviour Research and Therapy, 46(11), 1210-1219.

Robinson, TE, \& Berridge, KC. (1993). The neural basis of drug craving: An incentive-sensitization theory of addiction. Brain Research Reviews, 18(3), 247-291.

Rose, AK, Brown, K, Field, M, \& Hogarth, L. (2013). The contributions of value-based decision-making and attentional bias to alcohol-seeking following devaluation. Addiction, 108(7), 1241-1249.

Saunders, JB, Aasland, OG, Babor, TF, De la Fuente, JR, \& Grant, M. (1993). Development of the alcohol use disorders identification test (AUDIT): WHO collaborative project on early detection of persons with harmful alcohol consumption II. Addiction, 88(6), 791-804.

Sobell, LC, \& Sobell, MB. (1992). Timeline Follow-back: a technique for assessing self-reported ethanol consumption. In J Allen \& RZ Litten (Eds.), Measuring alcohol consumption: Psychosocial and biological methods (pp. 41-72). Totowa, NJ: Humana Press.

Spruyt, A, De Houwer, J, Tibboel, H, Verschuere, B, Crombez, G, Verbanck, P, \& Noël, X. (2013). On the predictive validity of automatically activated approach/avoidance tendencies in abstaining alcohol-dependent patients. Drug and Alcohol Dependence, 127(1-3), 81-86.

Stacy, AW, \& Wiers, RW. (2010). Implicit cognition and addiction: A tool for explaining paradoxical behavior. 6, 551-575.

Stormark, KM, Field, NP, Hugdahl, K, \& Horowitz, M. (1997). Selective processing of visual alcohol cues in abstinent alcoholics: An approach-avoidance conflict? Addictive Behaviors, 22(4), 509-519.

Stroebe, W, Mensink, W, Aarts, H, Schut, H, \& Kruglanski, AW. (2008). Why dieters fail: Testing the goal conflict model of eating. Journal of Experimental Social Psychology, 44(1), 26-36.

Stroebe, W, Van Koningsbruggen, GM, Papies, EK, \& Aarts, H. (2013). Why most dieters fail but some succeed: A goal conflict model of eating behavior. Psychological Review, 120(1), 110-138.

Townshend, JM, \& Duka, T. (2001). Attentional bias associated with alcohol cues: Differences between heavy and occasional social drinkers. Psychopharmacology, 157(1), 67-74.

Townshend, JM, \& Duka, T. (2007). Avoidance of alcohol-related stimuli in alcohol-dependent inpatients. Alcoholism: Clinical and Experimental Research, 31(8), 1349-1357.

Vakili, S, Sobell, LC, Sobell, MB, Simco, ER, \& Agrawal, S. (2008). Using the Timeline Followback to determine time windows representative of annual alcohol consumption with problem drinkers. Addictive Behaviors, 33(9), 1123-1130.

Van den Bussche, E, Van den Noortgate, W, \& Reynvoet, B. (2009). Mechanisms of Masked Priming: A Meta-Analysis. Psychological Bulletin, 135(3), 452-477.

Vollstädt-Klein, S, Loeber, S, Von der Goltz, C, Mann, K, \& Kiefer, F. (2009). Avoidance of alcohol-related stimuli increases during the early stage of abstinence in alcohol-dependent patients. Alcohol and Alcoholism, 44(5), 458-463

Watson, P, de Wit, S, Hommel, B, \& Wiers, RW. (2012). Motivational mechanisms and outcome expectancies underlying the approach bias toward addictive substances. Frontiers in Psychology. OCT.

Weafer, J, \& Fillmore, MT. (2013). Acute alcohol effects on attentional bias in heavy and moderate drinkers. Psychology of Addictive Behaviors, 27(1), 32-41.

Wentura, D, \& Degner, J. (2010). Automatic evaluation isn't that crude! moderation of masked affective priming by type of valence. Cognition and Emotion, 24(4), 609-628.

Wentura, D, Kulfanek, M, \& Greve, W. (2005). Masked affective priming by name letters: Evidence for a correspondence of explicit and implicit self-esteem. Journal of Experimental Social Psychology, 41(6), 654-663.

Wiers, RW, Bartholow, BD, van den Wildenberg, E, Thush, C, Engels, RCME, Sher, KJ, \& Stacy, AW. (2007). Automatic and controlled processes and the development of addictive behaviors in adolescents: A review and a model. Pharmacology, Biochemistry and Behavior, 86(2), 263-283.
Wiers, RW, Rinck, M, Dictus, M, \& Van Den Wildenberg, E. (2009). Relatively strong automatic appetitive action-tendencies in male carriers of the OPRM1 G-allele. Genes, Brain and Behavior, 8(1), 101-106.

Wiers, RW, Gladwin, TE, Hofmann, W, Salemink, E, \& Ridderinkhof, KR. (2013). Cognitive bias modification and cognitive control training in addiction and related psychopathology: Mechanisms, clinical perspectives, and ways forward. Clinical Psychological Science, 1(2), 192-212.

World Health Organisation. (1994). Lexicon of alcohol and drug terms. London.

\section{doi:10.1186/s40359-014-0028-1}

Cite this article as: Baker et al:: Implicit priming of conflicting motivational orientations in heavy drinkers. BMC Psychology 2014 2:28

\section{Submit your next manuscript to BioMed Central and take full advantage of:}

- Convenient online submission

- Thorough peer review

- No space constraints or color figure charges

- Immediate publication on acceptance

- Inclusion in PubMed, CAS, Scopus and Google Scholar

- Research which is freely available for redistribution

Submit your manuscript at www.biomedcentral.com/submit 